\title{
Nutrición, enfermedad ósea y cirrosis alcohólica
}

\author{
M. A. ESCALANTE BOLEAS, R. FRANCO VICARIO, L. CUBAS LARGACHA*, \\ J. I. GOIRÍA ORMAZÁBAL**, M. L. ZULUETA GABANCHO***, A. CABARCOS \\ CAZÓN****, C. DUQUE DE LAS HERAS***, F. MIGUEL DE LA VILLA \\ Servicio de Medicina Interna. Hospital de Basurto. *Centro de Cálculo. Universidad de \\ Deusto. **Área de Salud. Excmo. Ayuntamiento de Bilbao. ***Servicio de Bioquímica. \\ Hospital de Basurto. Bilbao. ****Servicio de Medicina Interna. Complejo Hospitalario \\ Donostia
}

\author{
NUTRITION, BONE DISEASE AND ALCOHOLIC CIRRHOSIS
}

\section{RESUMEN}

Objetivos: Conocer la prevalencia de osteopenia y osteoporosis en cirróticos alcohólicos entre 45 y 65 años de edad. Comprobar si el estado nutricional está implicado en la pérdida de masa ósea de la enfermedad hepática.

Material y métodos: Población a estudio: 52 varones cirróticos alcohólicos de 45 a 65 años de edad, con función renal normal, no sedentarios y sin ningún tratamiento que pudiera alterar el estudio. Diseño y variables: estudio descriptivo transversal finalizado en 2 años. El estado nutricional se evaluó por medio de parámetros antropométricos clásicos (peso, altura, perímetro braquial y pliegues cutáneos) y densitometría corporal total (absortimetría de doble haz de RX DPX plus LUNARDEXA). Análisis de los datos: ordenador Mc Intosh (programa Filemaker PRO). Se usó la t de Student para comparar grupos y p fue significativo si $\alpha \leq 0,05$. Gravedad clínica de la cirrosis: clasificación de Child-Turcotte.

Resultados: Se consideró osteopenia si la densidad mineral ósea (DMO) estaba por encima de 1 desviación estándar (DE) pero por debajo de $3 \mathrm{DE}$ respecto a la $\mathrm{T}$ score (valores promedio en mujeres adultas) y osteoporosis (OP) si la DMO estaba por encima de 3 DE o existían fracturas de compresión vertebrales.

La prevalencia de enfermedad ósea fue del $58 \%$.

Los porcentajes de grasa obtenidos por medidas antropométricas y densitométricas fueron superiores en los pacientes no osteopénicos.

Discusión: La prevalencia de enfermedad ósea fue superior a la de otros estudios.

La densitometría corporal total es un método más exacto para medir el porcentaje de grasa corporal. Los porcentajes de grasa obtenidos con ambos métodos no se relacionaron con la pérdida de masa ósea. El estado nutricional como factor aislado no conlleva desmineralización ósea en estos pacientes.

PALABRAS CLAVE: Cirrosis alcohólica. Estado nutricional. Enfermedad ósea.

\section{ABSTRACT}

Objectives: To know the prevalence of osteopenia and osteoporosis in alcoholic cirrhotic males from 45 to 65 years old. To prove if the nutritional state is involved in the loss of bone mass in the hepatic disea. se.

Material and methods: Study population: 52 alcoholic cirrhotic males from 45 to 65 years old, with normal kidney function, non-seden tary and with no treatment that could alterate the study. Design and variables: transversal descriptive study finished in 2 years. The nutritio nal state was evaluated by means of classic anthropometric parameters (weight, height, perimeter of the arm and skin folds) and total body densitometry (DPX plus LUNAR-DEXA with dual energy X-ray absorptio metry. Statistical analysis: Mc Intosh computer (Filemaker PRO pro gram). T Student was used to compare groups and $p$ was significant if alpha $\alpha \leq 0.05$. Clinical severity of cirrhosis: Child-Turcotte classification.

Results: Osteopenia was diagnosed if bone mineral density (BMD) was higher than 1 standard deviation (SD) but lower than $3 S D$ below $T$ score (mean values for adult women) and osteoporosis $(O P)$ if $B M D$ was higher than $3 S D$ or vertebral compression fractures appeared in simple radiographies.

The prevalence of bone disease was 58\%. The percentages of fat obtained by means of anthropometric and densitometric measures were higher in non-osteopenic patients.

Discussion: The prevalence of bone disease was higher than another studies.

The total body densitometry is a more exact method to measure the percentage of body fat. The percentages of fat obtained with both met hods were not related to the loss of bone mass. The nutritional state as an isolated factor does not lead to bone disease in these patients.

KEY WORDS: Alcoholic cirrhosis. Nutritional state. Bone disease.

Escalante Boleas MA, Franco Vicario R, Cubas Largacha L, Goiría Ormazábal JI, Zulueta Gabancho ML, Cabarcos Cazón A, Duque de las Heras C, Miguel de la Villa F. Nutrición, enfermedad ósea y cirrosis alcohólica. An Med Interna (Madrid) 2002; 19: 503-505.

\section{INTRODUCCIÓN}

La osteoporosis (OP) es una enfermedad metabólica ósea consistente en la disminución de la masa ósea, lo que puede derivar en la aparición de fracturas patológicas.

Las alteraciones óseas encontradas en la cirrosis hepática (OP, osteomalacia y anomalías en el metabolismo fosfocálcico) se denominan globalmente osteodistrofia hepática. La

Trabajo aceptado: 27 de mayo de 2002

Correspondencia: Miguel Angel Escalante Boleas. C/ Gregorio Uzquiano, 24, $1^{\circ}$ dcha. Portugalete (Vizcaya). 
mayoría de los estudios realizados hasta la fecha concluyen que la OP es la osteopatía más frecuente en las distintas afecciones hepáticas y estaría englobada en el grupo de las OP secundarias.

Algunos autores han afirmado que el estado nutricional influye en el desarrollo de la enfermedad ósea, siendo la obesidad protectora y la delgadez un factor que puede predisponer a poseer una menor masa ósea, sobre todo a nivel del hueso cortical.

La densitometría DEXA es un método de medición no sólo de la masa ósea sino también de la masa grasa, muscular y el porcentaje de grasa, lo que permite obtener unos resultados más fiables que los obtenidos por parámetros bioquímicos y antropométricos.

\section{MATERIAL Y MÉTODOS}

Población a estudio: recogida de la muestra: se ordenó a todos los varones cirróticos alcohólicos de 45 a 65 años pertenecientes a los archivos clínicos del Hospital de Basurto, obteniéndose un total de 194.

Comenzando por el último, se seleccionó 1 de cada 3 sujetos hasta conseguir un número total de 74 pacientes que aceptaran participar en el estudio.

Requisitos de selección: -varones cirróticos alcohólicos de 45 a 65 años; -valores normales de función renal; -no recibir tratamiento con vitamina $\mathrm{D}$, estrógenos, antiandrógenos, corticoides o calcio; -no ser sujetos sedentarios.

Los pacientes fueron informados por escrito sobre los objetivos y la estructura principal del trabajo y dieron consentimiento por escrito para participar en el mismo.

Diseño y variables: estudio descriptivo transversal desarrollado en 2 años (1994 y 1995). Se evaluó a cada individuo en el Servicio de Medicina Interna y se les realizó una historia clínica y una exploración física completas.

El estado nutricional se determinó por 2 métodos: medidas antropométricas clásicas: peso, altura, perímetro braquial y pliegues cutáneos (bicipital, tricipital, abdominal y subescapular) con el lipocalibre EIYOKEN TYPE. El porcentaje de grasa se calculó por la fórmula de Siri: $(4,95 / \mathrm{D}-4,5)$ x 100 , siendo $\mathrm{D}=1,1620-0,0700 \times \log$ suma de pliegues cutáneos (40-49 años) y $\mathrm{D}=1,1715-0,0779$ x log suma pliegues cutáneos (> 50 años).

Densitometría corporal total: se utilizó un densitómetro de doble haz de RX (DPX plus LUNAR-sistema DEXA) para localizar automáticamente 9 regiones anatómicas. Además, un analizador de cuerpo entero permitió cuantificar la grasa corporal tanto en porcentaje como en gramos.

Análisis estadístico: la base de datos se diseñó en un ordenador Mc Intosh con el programa Filemaker PRO. Los datos se prepararon en un ordenador PERFORMA 6200, usando el programa BB Edit Lite 3.0. Finalmente, las variables se estudiaron en un ordenador Mc Intosh con el programa Statview.

Tratamiento estadístico: se aplicó la t de Student para comparar grupos y se calculó p para cada prueba estadística. $\mathrm{p}$ fue significativa si alfa $<0=0,05$.

Gravedad clínica de la cirrosis: se utilizó la clasificación de Child-Turcotte, separando a los pacientes en 3 grupos (A, B y C) dependiendo de la gravedad de su hepatopatía (1-3).

\section{RESULTADOS}

Porcentajes de osteopenia y osteoporosis: la Fundación Europea de Osteoporosis y Enfermedad Ósea, la Fundación Nacional de Osteoporosis de los USA y el WHO (4) han establecido y aceptado 4 categorías de diagnóstico para las mujeres adultas

(T score): normal: densidad mineral ósea (DMO) no mayor a 1 desviación estándar (DE) por debajo de la T score. Masa ósea baja u osteopenia: DMO mayor a 1 pero inferior a 2,5 DE por debajo de la T score. Osteoporosis: DMO mayor a 2,5 DE. Osteoporosis severa o establecida: DMO mayor a 2,5 DE y 1 o más fracturas de compresión vertebrales.

En nuestra muestra establecimos el corte en $3 \mathrm{DE}$ debido a que la masa ósea de los varones es mayor a la de las mujeres y la $\mathrm{T}$ score determina los valores promedio en mujeres adultas.

El $58 \%$ de los pacientes perdieron masa ósea (40\% osteopenia y $18 \%$ OP) (Fig. 1).

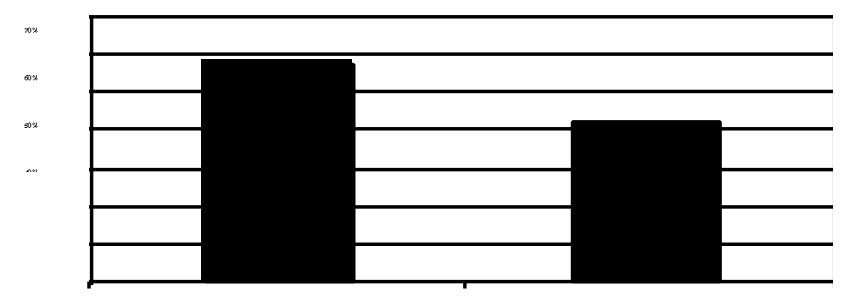

Fig. 1. Pérdida de masa ósea en la cirrosis alcohólica.

El 82,3\% de los sujetos presentaron pérdida de masa ósea en la columna lumbar y el 17,7\% en ambos cuellos femorales.

Estado nutricional y osteopenia: porcentaje de grasa (antropometría): la media de grasa fue $28,92 \%$ en los no osteopénicos y $27,69 \%$ en los sujetos osteopénicos y las DE = $3,005$ y 3,993 respectivamente. $\mathrm{p}$ ( t Student $)=0,26$.

Porcentaje de grasa (densitometría): las medias fueron $27,87 \%$ en los no osteopénicos y $26,75 \%$ en los pacientes osteopénicos y las DE =6,94 y 7,78. p = 0,60 (Fig. 2)

Gravedad clínica de la cirrosis: el $80,85 \%$ de los sujetos fueron Child A, 12,76\% Child B y 6,39\% Child C.

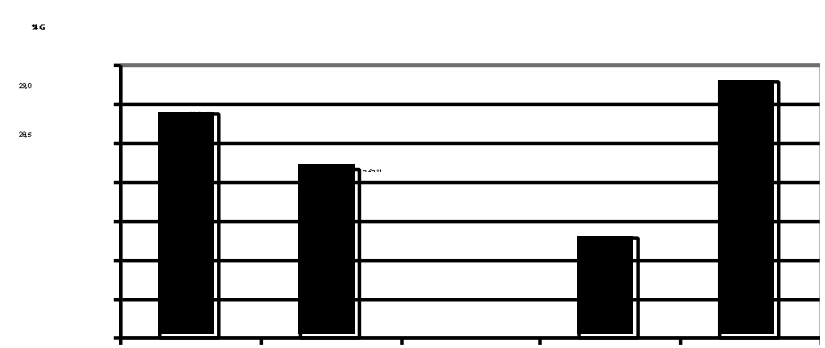

Fig. 2. 


\section{DISCUSIÓN}

La prevalencia de osteopenia en la cirrosis hepática y otras hepatopatías es variable.

Diamond y cols. obtuvieron porcentajes entre el 28-39\% en series de 40 a 115 pacientes (5-8).

Otros autores como Bonkovsky (9) y Rose trabajaron con series de 133 y 57 pacientes y obtuvieron prevalencias entre el 13 y $39 \%$ el primero y $23 \%$ el segundo.

Estas series estaban formadas por varones y mujeres de 20 a 74 años que sufrían enfermedades como hepatitis crónica activa, hemocromatosis, cirrosis biliar primaria, cirrosis alcohólica o colangitis esclerosante primaria. Todas estas patologías se englobaron en una acepción común: enfermedades hepáticas crónicas.

Por otra parte, Conte (11) y Mobarhan (12) estudiaron la osteopenia sólo en la cirrosis alcohólica, obviando la edad y el sexo, de modo que las muestras fuesen homogéneas y formadas únicamente por varones hasta 65 años con la cirrosis alcohólica como único factor de riesgo.

Mobarhan, en una muestra similar (56 pacientes) obtuvo una prevalencia inferior (32\%) a la nuestra $(58 \%)$.

La evaluación del estado nutricional requiere utilizar 3 tipos de parámetros antropométricos: pliegues cutáneos, circunferencia braquial y porcentaje de grasa corporal total calculado a partir de los pliegues cutáneos (13).

Si sólo se midiesen los pliegues cutáneos se podría clasificar erróneamente a un sujeto como desnutrido, aún siendo normales su circunferencia braquial y su área muscular (13).

Habitualmente se considera que hay desnutrición cuando el porcentaje de grasa es menor al $10 \%$ y obesidad si es superior al 35\% (los valores normales en hombres y mujeres son 20 y $30 \%$ respectivamente).

En nuestra muestra, el porcentaje de grasa determinado por las medidas antropométricas fue menor al $20 \%$ en 1 paciente y ninguno presentó un porcentaje de grasa superior al $35 \%$. Sin embargo, la densitometría detectó 8 sujetos con porcentajes inferiores al 20\% (1 de ellos tenía además osteopenia) y 7 con porcentajes superiores al $35 \%$ (3 pacientes tenían osteopenia).

Estos resultados nos sugieren que la densitometría es un método más preciso para medir el porcentaje de grasa corporal, ya que generalmente no está tan sujeto a variaciones intraobservador e intramétodo.

La media de grasa en los pacientes osteopénicos fue inferior a la de los no osteopénicos con ambos métodos. En la literatura se afirma que cuanto mayor sea el porcentaje de grasa corporal, mayor será la protección contra la pérdida de masa ósea.

Por lo tanto, parece que los resultados obtenidos están de acuerdo con esta última afirmación. No obstante, la relación osteopenia-estado nutricional no fue significativa desde el punto de vista estadístico.

No se ha podido demostrar que el estado nutricional como factor aislado tenga influencia sobre la pérdida de masa ósea en los sujetos de la muestra.

En estos momentos el debate está abierto. Si hubiésemos trabajado con una muestra con peor estado nutricional (Child $\mathrm{C}$, con niveles bajos de albúmina) quizás habríamos obtenido resultados diferentes, ya que esta proteína está relacionada con la nutrición de los pacientes (14).

\section{Bibliografía}

1. Chen CC, Wang SS, Heng FS, Lee SD. Metabolic bone disease of liver cirrhosis: is it parallel to the clinical severity of cirrhosis? J Gastroenterol Hepatol 1996; 11 (5): 417-21.

2. Conn HO. A peek at the Child-Turcotte classification. Hepatology 1981; 1: 673-6.

3. Child III GG, Turcotte JG. Surgery and portal hypertension. En Child III GG, ed. The liver and portal hypertension. Philadelphia, WB Saunders Co, 1964; 50.

4. Kanis JA. Osteoporosis y sus consecuencias. En: Kanis JA, ed. Osteoporosis $1^{a}$ ed (ed esp). Londres: Blackwell Science Ltd, 1996; 1-25.

5. Diamond T, Stiel D, Lunzer M, Wilkinson M, Posen S. Ethanol reduces bone formation and may cause osteoporosis. Am J Med 1989; 86: 2828.

6. Diamond T, Stiel D, Mason R, et al. Serum vitamin D metabolites are not responsible for low turnover in chronic liver disease. J Clin Endocrinol Metab 1989; 69 (6): 1234-9.

7. Diamond T, Stiel D, Lunzer M, Mc Dowall D, Eckstein RP, Posen S. Hepatic osteodystrophy. Static and dynamic bone histomorphometry and serum bone gla-protein in 80 patients with chronic liver disease. Gastroenterology 1989; 96 (1): 213-21.

8. Diamond T, Stiel D, Lunzer M, Wilkinson M, Roche J, Posen S. Osteoporosis and skeletal fractures in chronic liver disease. Gut 1990; 31: 82-

9. Bonkovsky ML, Hawkins M, Steinberg K, et al. Prevalence and prediction of osteopenia in chronic liver disease. Hepatology 1990; 12 (2): 273-80.

10. Rose JDR, Crawley EO, Evans WD, et al. Osteoporosis in chronic liver disease. Bone 1987; 8: 53.

11. Conte D, Caraceni MP, Duriez J, et al. Bone involvement in primary hemochromatosis and alcoholic cirrhosis. Am J Gastroenterol 1989; 84 (10): 1231-4.

12. Mobarhan SA, Russell RM, Recker RR, posner DB, Iber FL, Miller P Metabolic bone disease in alcoholic cirrhosis: a comparison of the effect of vitamin D2, 25-hydroxyvitamin D or supportive treatment. Hepatology 1984; 4 (2): 266-73.

13. Alastrue A, Sitges A, Jaurrieta E, Puig P, Abad JM, Sitges A. Valoración antropométrica del estado de nutrición: normas y criterios de desnutrición y obesidad. Med Clin (Barc) 1983; 80 (16): 691-9.

14. Dionigi R, Dominioni L, Jemos V, Cremaschi R, Monico R. Diagnosing malnutrition. Gut 1986; 27 (51): 5-8. 